\title{
AN IMPLICIT FUNCTION THEOREM FOR NONDIFFERENTIABLE MAPPINGS
}

\author{
SHUI-NEE CHOW ${ }^{1}$ AND A. LASOTA ${ }^{2}$
}

\begin{abstract}
By using Borsuk's antipodal theorem, an implicit function theorem for nondifferentiable mappings in Banach spaces is proved. Applications of this theorem to give existence and continuous dependence on a parameter of solutions of certain boundary value problems, are shown.
\end{abstract}

1. Introduction. It is well known that implicit function theorems enable us to obtain existence theorems for periodic solutions and solutions of boundary value problems for ordinary differential equations. Generally implicit function theorems are not related to the compactness of operators, since they can be proved by Banach's contraction principle. However, operators defined by ordinary differential equations are generally compact. It is this fact that motivates us to replace the usual implicit function theorem by a different one which is not related to Banach's contraction principle, but to Borsuk's antipodal fixed point theorem.

Our implicit function theorem will be stated by using "multivalued derivative", a concept which is very similar to the one used in [1] and [2].

Existence of periodic solutions and solutions of boundary value problems will be shown by applying the multivalued implicit function theorem. Moreover, this theorem will give us a family of solutions which have the continuous dependence property.

2. Implicit function theorem. Let $E$ be a Banach space with norm $\mid \cdot$. Let $\operatorname{cf}(E)$ denote the set of all nonempty closed convex subsets of $E$. If $A \in \operatorname{cf}(E),|A|=\sup \{|x|: x \in A\}$. Throughout this section, $U$ will denote an open subset of $E$.

DEFINITION 1. A map $P: E \rightarrow \operatorname{cf}(E)$ is called homogeneous if for every real $\lambda, P(\lambda x)=\lambda P(x)$, for all $x \in E$.

Received by the editors July 2, 1971 and, in revised form, October 21, 1971.

AMS 1970 subject classifications. Primary 34A10; Secondary 34B10, 34C25.

Key words and phrases. Implicit function theorem, multivalued derivatives, completely continuous mappings, Borsuk antipodal theorem, boundary value problems, Nicoletti problem, aperiodic boundary condition.

${ }^{1}$ Michigan State University. Partially supported by NSF GU-2648.

${ }^{2}$ Jagellonian University, Krakow, Poland.

(C) American Mathematical Society 1972 
Definition 2. Let $p: U \rightarrow E$, and $x_{0} \in U$. A homogeneous map $P: E \rightarrow$ $\operatorname{cf}(E)$ is called an upper derivative of $p$ at $x_{0}$ if $p(x)-p\left(x_{0}\right) \in P\left(x-x_{0}\right)+$ $a\left(\left|x-x_{0}\right|\right)$ where $\left|a\left(\left|x-x_{0}\right|\right)\right| /\left|x-x_{0}\right| \rightarrow 0$ as $x \rightarrow x_{0}$.

Definition 3. A map $P: E \rightarrow \operatorname{cf}(E)$ is called completely continuous if

(1) it is upper semicontinuous, i.e., the graph $\{(x, y): y \in P(x)\}$ is closed in $E \times E$; and

(2) it is compact, i.e., for every bounded subset $V \subset E$, the set $\{y: y \in P(x), x \in V\}$ has compact closure.

Let $V$ be a normed space. The ball in $V$ with radius $\varepsilon$ and center 0 will be denoted by $B(\varepsilon)$. We now are able to state our main theorem.

THEOREM 1. Given a functional equation,

$$
x=p(x)+q(x, v)
$$

where $p: U \rightarrow E$ and $q: U \times B(\alpha) \rightarrow E$ are completely continuous, $\alpha>0$, and $q(x, v) \rightarrow 0$ uniformly in $x$ as $|v| \rightarrow 0$. Suppose there exists $x_{0} \in U$, such that $x_{0}=p\left(x_{0}\right)$, and that there exists an upper derivative $P$ of $p$ at $x_{0}$, such that $P$ is completely continuous, and $x \in P(x) \Rightarrow x=0$. Then there exist $\bar{\varepsilon} \in(0, \alpha]$ and $R>0$, such that,

(1) for every $v \in B(\bar{\varepsilon})$, there is a solution $x_{v}$ of equation (*), which satisfies $\left|x_{v}-x_{0}\right|<R ;$ and

(2) $\left|x_{v}-x_{0}\right| \rightarrow 0$ as $|v| \rightarrow 0$.

LEMMA 1. Under the conditions of Theorem 1, there exists $J>0$, such that

$$
x \in P(x)+b \Rightarrow|x| \leqq J|b| .
$$

Proof. For if not, there exists a sequence $\left\{x_{n}\right\}$ satisfying $x_{n} \in P\left(x_{n}\right)+$ $b_{n}$, and $\left|x_{n}\right| \geqq n\left|b_{n}\right|$. Since $P$ is homogeneous, we have

$$
x_{n} /\left|x_{n}\right| \in P\left(x_{n} /\left|x_{n}\right|\right)+b_{n} /\left|x_{n}\right| .
$$

It follows from the compactness of $P$ and the inequality $\left|b_{n}\right| /\left|x_{n}\right| \leqq 1 / n$ that there exists $y \in E$, such that it is the limit of a subsequence of $\left\{\left|x_{n}\right|^{-1} x_{n}\right\}$. The upper semicontinuity of $P$ ensures that $y \in P(y),|y|=1$. This contradicts the assumption.

Proof of Theorem 1. Let $J$ be as in Lemma 1 , and let $R>0$ satisfy

$$
\left|a\left(\left|x-x_{0}\right|\right)\right| /\left|x-x_{0}\right|<1 / 2 J, \text { for all }\left|x-x_{0}\right|<R .
$$

From the conditions on $q(x, v)$, we may assume that there exists a monotone function $\delta=\delta(\cdot) \geqq 0$ such that $\delta(r) \rightarrow 0$ as $r \rightarrow 0$ and

$$
|q(x, v)|<\delta(|v|) \text { for all }\left|x-x_{0}\right|<R .
$$


Suppose $x_{v}$ is a solution of $(*)$ and $\left|x_{v}-x_{0}\right|<R$, then

$$
\begin{aligned}
x_{v}-x_{0} & =p\left(x_{v}\right)-p\left(x_{0}\right)+q\left(x_{v}, v\right) \\
& \in P\left(x_{v}-x_{0}\right)+a\left(\left|x_{v}-x_{0}\right|\right)+q\left(x_{v}, v\right) .
\end{aligned}
$$

Therefore from Lemma 1,

$$
\left|x_{v}-x_{0}\right| \leqq J\left(\left|a\left(\left|x_{v}-x_{0}\right|\right)\right|+\delta(|v|)\right) \leqq J\left((1 / 2 J)\left|x_{v}-x_{0}\right|+\delta(|v|)\right) .
$$

Hence, $\left|x_{v}-x_{0}\right|<J \delta(|v|)$. This proves the second part of the theorem.

Now let

and

$$
\begin{aligned}
\bar{p}(u) & =p\left(x_{0}+u\right)-p\left(x_{0}\right)=p\left(x_{0}+u\right)-x_{0}, \\
\bar{q}(u) & =q\left(x_{0}+u, v\right),
\end{aligned}
$$

$$
\varphi_{\imath}(u)=u-\bar{p}(u)-\bar{q}(u) .
$$

We claim that, for every $v \in B(\bar{\varepsilon})$,

$$
\varphi_{v}(u) \neq \lambda \varphi_{v}(-u), \quad \text { for all } \lambda \in[0,1],|u|=K,
$$

where $K=2 J \delta(|v|)$ and $\bar{\varepsilon}$ is so chosen that for every $v \in B(\bar{\varepsilon})$, we have $K<R$. Suppose not; then there exist $v \in B(\bar{\varepsilon})$ and $\lambda \in[0,1]$ such that $\varphi_{v}(u)=$ $\lambda \varphi_{v}(-u)$, for some $u$ satisfying $|u|=K<R$. Hence

$$
u=\frac{1}{1+\lambda} \bar{p}(u)+\frac{\lambda}{1+\lambda}(-\bar{p}(-u))+\frac{1}{1+\lambda} \bar{q}(u)+\frac{\lambda}{1+\lambda}(-\bar{q}(-u)) .
$$

It is not difficult to see that $-\bar{p}(-u) \in P(u)+a(|u|)$. Therefore, from the convexity of $P$, we have

$$
u \in P(u)+a(|u|)+\frac{1}{1+\lambda} \bar{q}(u)+\frac{\lambda}{1+\lambda}(-\bar{q}(-u)) .
$$

From Lemma 1, we obtain

$$
|u| \leqq J(|a(|u|)|+\delta(|v|))<\frac{1}{2}|u|+J \delta(|v|) .
$$

Hence $|u|<2 J \delta(|v|)=K$. This contradicts our assumption and hence proves the claim. Now by applying Borsuk's antipodal fixed point theorem $\left[3\right.$, p. 59] to the mapping $\varphi_{v}(u)$, we obtain $u_{v}$ such that $\left|u_{v}\right|<R$ and $\varphi_{v}\left(u_{v}\right)=0$. Setting $x_{0}+u_{v}=x_{v}$, we have the first part of Theorem 1.

3. Boundary value problem. Let $\Delta=[0,1]$, and $C$ be the space of all continuous functions $x: \Delta \rightarrow R^{n}$ with the usual norm topology, $|x|=$ $\sup \{|x(t)|: t \in \Delta\}$.

Consider the boundary value problems on $\Delta$,

$$
\begin{aligned}
& \dot{x}(t)=f(t, x(t)), \quad L_{0} x=r_{0}, \\
& \dot{x}(t)=f(t, x(t))+g(t, x(t), \varepsilon), \quad L_{\varepsilon} x=r_{\varepsilon},
\end{aligned}
$$


where $x(t) \in R^{n}, L_{\varepsilon}: C \rightarrow R^{n}$ is continuous and linear, $\varepsilon$ is a real parameter, and $r_{\varepsilon} \in R^{n}$. Let $x_{0}(\cdot)$ be a solution of (1) and $U=\{(t, x):|x-\bar{x}(t)|<1\} \subset$ $\Delta \times R^{n}$. We shall assume that

(i) $f: U \rightarrow R^{n}$ and $g: U \times[-\alpha, \beta] \rightarrow R^{n}$ are continuous, and there exists $M>0$ such that $|f(t, x)-f(t, y)| \leqq M|x-y|$, for all $(t, x) \in U$,

(ii) the map $\varepsilon \rightarrow L_{\varepsilon}$ is continuous.

THEOREM 2. If the boundary value problem

$$
|\dot{x}(t)| \leqq M|x(t)|, \quad L_{0} x=0,
$$

admits only the zero solution, then there exists $\bar{\varepsilon}>0$, such that for every $\varepsilon$ with $|\varepsilon|<\bar{\varepsilon}$, there is a solution $x_{\varepsilon}(\cdot)$ of $(2)$ and $x_{\varepsilon}(t) \rightarrow x_{0}(t)$ uniformly in $t$ as $\varepsilon \rightarrow 0$.

Proof. We note that if

and

$$
(p(x))(t)=x(0)+\int_{0}^{t} f(s, x(s)) d s+L_{0} x-r_{0}
$$

$$
(q(x, \varepsilon))(t)=\int_{0}^{t} g(s, x(s), \varepsilon) d s+L_{\varepsilon} x-r_{\varepsilon}-\left(L_{0} x-r_{0}\right),
$$

then problems (1) and (2) are equivalent to equation $(*)$ when $\varepsilon=0$ and $\varepsilon \neq 0$ respectively. By using the techniques in [4], it can be observed that all conditions in Theorem 1 on $p$ and $q$ are satisfied. The multivalued mapping,

$$
(P(x))(t)=\left\{x(0)+\int_{0}^{t} u(s) d s+L_{0} r: u(s) \in F(s, x(s))\right\}
$$

where $F(s, x(s))=\left\{y \in R^{n}=|y| \leqq M|x(s)|\right\}$ can also be shown by the methods in [5], to satisfy the conditions in Theorem 2 . With these observations, the conclusions of the theorem follow from Theorem 1.

4. Some applications. (1) Consider the Nicoletti problem [6]

$$
\begin{aligned}
\dot{x}_{i}(t) & =f_{i}\left(t, x_{1}(t), \cdots, x_{n}(t)\right), \\
x_{i}\left(t_{i}\right) & =r_{i}, \quad 0 \leqq t_{1} \leqq \cdots \leqq t_{n} \leqq 1,
\end{aligned}
$$

where $x_{i}$ 's and $r_{i}$ 's are real. Let $\tau=\left(t_{1}, \cdots, t_{n}\right)$. We note that Theorem 2 holds true when the parameter $\varepsilon$ is in $R^{n}$. Hence as a consequence of Theorem 2, we have

THEOREM 3. If $f_{i}$ 's are Lipschitzian in $\left(x_{1}, \cdots, x_{n}\right)$, and if $|\tau|=$ $\left|t_{1}\right|+\cdots+\left|t_{n}\right|$ is sufficiently small, then the Nicoletti problem is always solvable. Moreover, $x_{i, \tau}(t) \rightarrow x_{i .0}(t)$ uniformly in $t$ as $|\tau| \rightarrow 0$, where $\left(x_{1,0}(t), \cdots, x_{n, 0}(t)\right)$ is the solution of (4) for the Cauchy problem $\left(x_{1}(0), \cdots, x_{n}(0)\right)=\left(r_{1}, \cdots, r_{n}\right)$. 
(2) Consider the aperiodic boundary value problem $(\lambda>0 ; x=$ $\left.\left(x_{1}, \cdots, x_{n}\right) \in R^{n} ; \varepsilon \in R\right)$ :

$$
\dot{x}=f(t, x)+\varepsilon g(t, x, \varepsilon), \quad x(0)+\lambda x(1)=0 .
$$

The following lemma is in [7].

LEMMA 2. If $b(t) \geqq 0$, and

$$
\int_{0}^{1} b(t) d t<\left(\pi^{2}+\log ^{2} \lambda\right)^{1 / 2}
$$

then $x(t) \equiv 0$ is the unique solution for

$$
|\dot{x}(t)| \leqq b(t)|x(t)|, \quad x(0)+\lambda x(1)=0 .
$$

From Theorem 2 and Lemma 2 there follows immediately

THEOREM 4. Let $f$ and $g$ be continuous, and let $|f(t, x)-f(t, y)| \leqq$ $b(t)|x-y|$, where $b(\cdot)$ satisfies (7). Suppose $x_{0}(\cdot)$ is the solution of (6) for $\varepsilon=0$, then there exists $\bar{\varepsilon}>0$ such that for every $\varepsilon$ with $|\varepsilon|<\bar{\varepsilon}$, there is a solution $x_{\varepsilon}(\cdot)$ of $(6)$ and $x_{\varepsilon}(t) \rightarrow x_{0}(t)$ uniformly in $t$ as $\varepsilon \rightarrow 0$.

(3) Observe that under the assumptions of Theorem 3, equation (4) may not be locally linearized. Therefore the usual implicit function theorem is not applicable. The same comments are valid for Theorem 4 . The purpose of stating those problems is to show the possibility of applications. It seems that many other results may be obtained in the same way. In particular, the continuous dependence theorems quoted in [8] may be generalized.

5. Remarks. (1) The condition: $x \in P(x) \Rightarrow x=0$ in Theorem 1 is analogous to the requirement that a Jacobian be nonzero.

(2) The following Borsuk's theorem is included for the convenience of the reader.

THEOREM. Let $X=\{u \in E:|u| \leqq K\}$ be a ball with radius $K$ in $E$, and $P: X \rightarrow E$ be completely continuous. Suppose $\varphi(u)=u-p(u)$, then either there exist $\lambda \in[0,1]$, and $u \in X$ such that $\varphi(u)=\lambda \varphi(-u)$, and $|u|=K$ or there exists $u \in X$ such that $\varphi(u)=0$.

\section{REFERENCES}

1. A. Lasota and A. Strauss, Asymptotic behavior for differential equations which cannot be locally linearized, J. Differential Equations 10 (1971), 152-172.

2. Shui-Nee Chow, L. Karlovitz and A. Lasota, An integral form of the mean value theorem for non-differentiable mappings, J. Math. Anal. Appl. (to appear).

3. A. Granas, Introduction to topology of functional spaces, Math. Lecture Notes, University of Chicago, Chicago, Ill., 1961. 
4. M. A. Krasnosel'skiir, Topological methods in the theory of nonlinear integral equations, GITTL, Moscow, 1956; English transl., Macmillan, New York, 1964. MR 20 \#3464; MR 28 \#2414.

5. A. Lasota and Z. Opial, An application of the Kakutani-Ky Fan theorem in the theory of ordinary differential equations, Bull. Acad. Polon. Sci. Sér. Sci. Math. Astronom. Phys. 13 (1965), 781-786. MR 33 \#4370.

6. $O$. Nicoletti, Sulle condizioni iniziali che determinano gli integrali delle equazioni differenziali ordinaire, Atti. Accad. Sci. Torino 33 (1897/98), 746-759.

7. S. Kasprzyk and J. Myjak, On the existence of solutions of the Floquet problem for ordinary differential equations, Zeszyty Nauk. Uniw. Jagiello. Prace Mat. Zeszyt 13 (1969), 35-39. MR 40 \#441.

8. A. Lasota and Z. Opial, Sur la dépendance continue des solutions des équations différentielles ordinaires de leurs seconds membres et des conditions aux limites, Ann. Polon. Math. 19 (1967), 13-36. MR 34 \#7852.

Department of Mathematics, Michigan State University, East lansing, MiCHIGAN 48823

Institute of Mathematics, Jagellonian University, Krakow, Poland 